\title{
Microscopic calculations of weak decays in superheavy nuclei
}

\author{
P. Sarriguren* \\ Instituto de Estructura de la Materia, IEM-CSIC, Serrano 123, E-28006 Madrid, Spain
}

(Dated: July 17, 2019)

\begin{abstract}
Half-lives of $\beta^{+}$decay and electron capture are studied in some selected superheavy nuclei produced in hot-fusion reactions, namely, ${ }^{290} \mathrm{Fl},{ }^{293} \mathrm{Mc},{ }^{294} \mathrm{Lv}$, and ${ }^{295} \mathrm{Ts}$. The nuclear structure is described microscopically from deformed self-consistent Skyrme Hartree-Fock mean-field calculations that include pairing correlations. The sensitivity of the half-lives to deformation and to the $Q_{E C}$ energies, which are still not determined experimentally, are studied. The results are compared with phenomenological $\alpha$-decay half-lives, showing that the latter decay mode is dominant in this mass region.
\end{abstract}

\section{INTRODUCTION}

The search for new regions of nuclear stability in superheavy nuclei (SHN) is a very active and successful line of research that has already led to the discovery of a large number of new elements [1-8]. First calculations of binding energies within macroscopic-microscopic models [8 14] predicted the existence of "islands of stability" for spherical SHN $Z=114$ and $N=184$, as well as for deformed nuclei with $Z=108$ and $N=162$. In these models a macroscopic term, usually derived from a deformed liquid-drop model, is complemented with a microscopic part that includes a shell correction derived from a shell model calculation. Purely microscopic calculations showed that the location of the shell closures in SHN is not very robust, but model dependent. Different selfconsistent relativistic and non-relativistic meanfield models [15-20] predict closure of spherical shells at $Z=114, N=184, Z=120, N=172$, and $Z=126, N=184$, depending on the interactions and their parametrizations. Note that the macroscopicmicroscopic calculations performed with the modified two-center shell model [21] reveal quite strong shell effects at $Z=120-126$ and $N=184$, in agreement with the self-consistent mean-field treatments.

In parallel, different experimental strategies were successfully carried out to reach the theoretically predicted "islands of stability" for SHN. The cold-fusion approach was used to synthesize SHN with $Z=107-112$ in reactions with target magic nuclei $\left({ }^{208} \mathrm{~Pb}\right.$ and $\left.{ }^{209} \mathrm{Bi}\right)$ and massive projectiles, such as ${ }^{50} \mathrm{Ti},{ }^{54} \mathrm{Cr},{ }^{58} \mathrm{Fe},{ }^{62,64} \mathrm{Ni}$, and ${ }^{70} \mathrm{Zn} \mathrm{[5].} \mathrm{These} \mathrm{reactions} \mathrm{are} \mathrm{cold} \mathrm{in} \mathrm{the} \mathrm{sense} \mathrm{that} \mathrm{the}$ compound nucleus has low excitation energy and only one or two neutrons evaporate. However, the method is not applicable for reaching heavier nuclei, in particular around $Z=114, N=184$, because of the fast decrease of the production cross sections for increasing charge of the projectile. To overcome this difficulty, a second strategy was developed, using more asymmetric reactions (less Coulomb repulsion) with both target and projectile hav-

*Electronic address: p.sarriguren@csic.es ing a large neutron excess. Following this strategy, longlived actinide nuclei from ${ }^{238} \mathrm{U}$ to ${ }^{249} \mathrm{Cf}$ were used as targets, whereas the double magic nucleus ${ }^{48} \mathrm{Ca}$ was used as a beam. These so called hot-fusion reactions result in the production of SHN with $Z=112-118$ in the neutronevaporation channels ( $x n$-channels) [4]. The main advantage of these reactions is that the Coulomb force becomes weaker as compared to the cold-fusion reactions and the probability of forming a compound nucleus increases dramatically. However, in hot-fusion reactions the compound nucleus formed is highly excited and more neutrons are evaporated. After evaporation of $x$ neutrons $(x=2-5)$, nearly all the new nuclei produced in the hotfusion reactions undergo a chain of $\alpha$ decays ending with a spontaneous fission. Identification of the associated $\alpha$ decay chains is the link to establish the original SHN.

Further experimental extension of the SHN region in the direction of the magic neutron number $N=184$, where the center of the "island of stability" is predicted, is limited in the $x n$-channels by the number of available stable projectiles and targets and the small production cross sections. One possible alternative would be to exploit reactions with neutron-rich radioactive beams. Because the intensive radioactive beams are not available so far, new isotopes of heaviest nuclei with $Z=111-117$ can be synthesized in the ${ }^{48} \mathrm{Ca}$-induced actinide-based complete fusion-evaporation reactions with the emission of charged particles ( $p x n-$ and $\alpha x n$-channels) from the compound nucleus [6]. The evaporation of proton or $\alpha$-particle from compound nucleus in these reactions, for example ${ }^{48} \mathrm{Ca}+{ }^{248} \mathrm{Cm} \rightarrow{ }^{290} \mathrm{Fl}+\alpha 2 n$, ${ }^{48} \mathrm{Ca}+{ }^{248} \mathrm{Cm} \rightarrow{ }^{293} \mathrm{Mc}+p 2 n, \quad{ }^{48} \mathrm{Ca}+{ }^{249} \mathrm{Bk} \rightarrow{ }^{294} \mathrm{Lv}+p 2 n$, ${ }^{48} \mathrm{Ca}+{ }^{251} \mathrm{Cf} \rightarrow{ }^{295} \mathrm{Ts}+p 3 n$, leads to the formation of nuclei with smaller $Z$, but with larger neutron excess. In addition, in the nucleus formed the electron capture (EC) can occur by converting a proton into a neutron to the daughter nucleus. Therefore, it is of great interest to study the competition between $\beta^{+} / \mathrm{EC}$ and $\alpha$ decays in SHN produced in the $p x n$ and $\alpha x n$ evaporation channels of hot-fusion reactions.

In addition, the $\beta^{+}$decay and EC branches would open the possibility to reach other SHN not belonging the original $\alpha$-decay chains. These new branches would be open if $\beta^{+} / \mathrm{EC}$ and $\alpha$-decay half-lives are comparable [22, 23]. In Ref. [8], the competition between $\beta^{+} / \mathrm{EC}$ and $\alpha$ decays 
has been considered in ${ }^{290} \mathrm{Fl}$, arguing about the possibility of populating a new $\alpha$-decay chain started at ${ }^{290} \mathrm{Nh}$. So, the study of $\beta^{+} / \mathrm{EC}$ decay modes is also important for the unambiguous identification of new SHN.

In this paper the focus of attention is the $\beta^{+} / E C$ decay mode in SHN that so far has been studied only at a phenomenological level 22 25]. In this work the $\beta^{+} / E C$ decay half-lives are calculated microscopically from an effective nucleon-nucleon interaction within a deformed selfconsistent mean-field Hartree-Fock calculation with Skyrme forces and pairing correlations in the BCS approximation. Four SHN are selected as representative of this mass region, namely, the isotopes of Flerovium $(Z=114, N=176),{ }^{290} \mathrm{Fl} ; \operatorname{Moscovium}(Z=115$, $N=178),{ }^{293} \mathrm{Mc}$; Livermorium $(Z=116, N=178)$, ${ }^{294} \mathrm{Lv}$; and Tennessine $(Z=117, N=178),{ }^{295} \mathrm{Ts}$.

The structure of the paper is as follows. I first review briefly in Section II the theoretical method used to calculate Gamow-Teller (GT) strength distributions and $\beta^{+} / E C$ half-lives. Then, I proceed to show the results in Section III. In Section III.A the ability of the method to reproduce the half-lives of nuclei is tested in the vicinity of $Z=100$, where experimental data are available. Section III.B contains the results for the SHN mentioned above. Finally, Section IV contains the summary and conclusions.

\section{THEORETICAL FRAMEWORK}

The $\beta^{+} / E C$-decay half-life, $T_{\beta+/ E C}$, is calculated by summing all the allowed GT transition strengths to states in the daughter nucleus with excitation energies $E_{e x}$, lying below the corresponding $Q_{i}$ energy $\left(i=\beta^{+}, E C\right)$,

$$
Q_{E C}=Q_{\beta^{+}}+2 m_{e}=M(A, Z)-M(A, Z-1)+m_{e},
$$

written in terms of the nuclear masses $M(A, Z)$ and the electron mass $\left(m_{e}\right)$. The GT strength is weighted with phase-space factors $f^{i}\left(Z, W_{0}\right)$, where the energy is $W_{0}=$ $Q_{i}-E_{e x}$.

$$
T_{i}^{-1}=\frac{\left(g_{A} / g_{V}\right)_{\mathrm{eff}}^{2}}{D} \sum_{E_{e x}<Q_{i}} f^{i}\left(Z, W_{0}\right) B\left(G T, E_{e x}\right),
$$

with $D=6143 \mathrm{~s}$ and $\left(g_{A} / g_{V}\right)_{\mathrm{eff}}=0.77\left(g_{A} / g_{V}\right)_{\text {free }}$, where 0.77 is a standard quenching factor and $\left(g_{A} / g_{V}\right)_{\text {free }}=-1.270$. Forbidden transitions are in general much smaller and therefore, they can be safely neglected, especially in nuclei with small $Q_{i}$-energies, such as those studied here.

Therefore, in the calculations of the $\beta^{+} / E C$ half-lives there are three main ingredients: (i) the $Q_{i}$ energies (maximum energy available in the process), which are taken from experiment when available or from different mass formulas or microscopic calculations in other cases; (ii) the phase-space factors for each transition, which are calculated in a similar way in practically all the existing calculations of the half-lives; and (iii) the nuclear structure that generates the energy distribution of the GT strength. This distribution may differ much among different approaches, such as simple constant values [22], distributions calculated with phenomenological potentials [25], or microscopic calculations based on effective nucleon-nucleon interactions like the work presented in this paper. I specify in what follows how these three pieces are treated.

Some recent calculations of half-lives in SHN by Karpov et al. [22] assume that the decay can be approximated by considering allowed transitions from the ground state of the parent nucleus to the ground state of the daughter. $Q_{i}$ energies are taken from the masses of the finite range droplet model (FRDM) [26] and the nuclear matrix elements of the transitions are assumed to be constant with $\log (f t)=4.7$ for all nuclei. The latter assumption might be very rough because it neglects any nuclear structure effect. It could be a large estimate of the average GT strength that finally would lead to half-lives being underestimated. In an older paper, 27] the authors used the same approach, but with $\log (f t)=6.5$. Then, in those references, only the phase-space factors remain to be calculated. $\beta^{+} / E C$ half-lives were also evaluated within a proton-neutron quasiparticle random phase approximation (pnQRPA) approach, which is based on a phenomenological folded-Yukawa single-particle Hamiltonian [25], using masses from FRDM and similar phase factors. Unfortunately, only $\beta^{+} / E C$ half-lives smaller than $100 \mathrm{~s}$ were published and the isotopes studied here are not in this category.

In the calculations of this work, the nuclear structure is described microscopically from selfconsistent deformed Hartree-Fock calculations with Skyrme forces and pairing correlations. The $Q_{i}$ energies in the cases where the masses are not measured are taken from different mass formulas that include masses from FRDM also used in the above references [22, 25]. The calculation of the phase factors is similar to those. Therefore, the current calculations represent an improvement over the previous ones with respect to the nuclear structure involved in the decay process.

\section{A. Mean-field approach for nuclear structure}

A brief summary of the theoretical formalism used in this paper to describe the nuclear structure involved in the $\beta^{+} / E C$-decay is presented here. Further details can be found elsewhere 28 31]. The starting point is a selfconsistent calculation of the mean field in terms of a deformed Hartree-Fock with Skyrme interactions and pairing correlations in the BCS approximation. The Skyrme interaction SLy4 [32] is selected because of its ability to account successfully for a large variety of nuclear properties all along the nuclear chart [33, 34]. Single-particle energies, wave functions, and occupation amplitudes are generated in this way. The solution of the HF equations 
is found by using the formalism developed in Ref. [35], assuming time reversal and axial symmetry. The singleparticle wave functions are expanded into the eigenstates of a harmonic oscillator with axial symmetry in cylindrical coordinates, using 16 major shells. It is well known that the harmonic oscillator basis used in the expansion of the deformed Hartree-Fock wave functions exhibits a Gaussian behavior at large distances that does not take properly into account effects of the continuum. These effects may be important in nuclei close to the drip lines. In mean-field approaches, this problem is cured by using a coordinate representation or a transformed harmonic oscillator basis that allows one to use a configuration space with the correct exponential asymptotic behavior [36]. Nevertheless, continuum effects are not expected to play any important role in the SHN studied in this work, which are close to islands of stability, and therefore they can be safely neglected here.

In the mean-field approach, the energy of the different shape configurations can be evaluated with constrained calculations, minimizing the Hartree-Fock energy under the constraint of keeping fixed the nuclear quadrupole deformation. The resulting total energy plots versus deformation are called in what follows deformation-energy curves (DEC). Deformation has been shown to be a key ingredient to understand the decay properties of $\beta$ unstable nuclei [28 31] and this would be of special importance in SHN.

In the next step, the GT strengths are calculated for the equilibrium shapes of each nucleus, that is, for the minima obtained in the DECs. Since decays connecting different shapes are disfavored, similar shapes are assumed for the ground state of the parent nucleus and for all populated states in the daughter nucleus [37-39].

To describe GT transitions, a deformed pnQRPA 2831, 37-40] formalism with spin-isospin residual interactions is used. However, in SHN the coupling strengths of these interactions are expected to be very small because they scale with the inverse of the mass number and therefore pnQRPA correlations are not expected to be especially relevant here, in particular for the half-lives that are only sensitive to the low energy region below the $Q$ window. Then, they are neglected in this work. Anyhow, the inclusion of pnQRPA correlations would result in a small reduction of the GT strength that would translate into a small increase of the corresponding half-lives.

The GT transition amplitudes in the intrinsic frame connecting the ground state $\left|0^{+}\right\rangle$of an even-even nucleus to one phonon states with energy $\omega_{K}$ in the daughter nucleus $\left|\omega_{K}\right\rangle(K=0,1)$ are found to be 28 31, 37 40],

$$
\left\langle\omega_{K}\left|\sigma_{K} t^{+}\right| 0\right\rangle=\sum_{\pi \nu}\left(\tilde{q}_{\pi \nu} X_{\pi \nu}^{\omega_{K}}+q_{\pi \nu} Y_{\pi \nu}^{\omega_{K}}\right),
$$

with

$$
\tilde{q}_{\pi \nu}=u_{\nu} v_{\pi} \Sigma_{K}^{\nu \pi}, \quad q_{\pi \nu}=v_{\nu} u_{\pi} \Sigma_{K}^{\nu \pi},
$$

in terms of the occupation amplitudes for neutrons and protons $v_{\nu, \pi}\left(u_{\nu, \pi}^{2}=1-v_{\nu, \pi}^{2}\right)$ and the matrix elements of the spin operator, $\Sigma_{K}^{\nu \pi}=\left\langle\nu\left|\sigma_{K}\right| \pi\right\rangle$, connecting proton and neutron single-particle states, as they come out from the HF+BCS calculation. $X_{\pi \nu}^{\omega_{K}}$ and $Y_{\pi \nu}^{\omega_{K}}$ are the forward and backward amplitudes of the pnQRPA phonon operator, respectively.

Once the intrinsic amplitudes in Eq. (3) are calculated, the GT strength $B\left(G T^{+}\right)$in the laboratory system for a transition $I_{i} K_{i}\left(0^{+} 0\right) \rightarrow I_{f} K_{f}\left(1^{+} K\right)$ can be evaluated. Using the Bohr-Mottelson factorization [41] to express the initial and final states in the laboratory system in terms of intrinsic states, one arrives at

$$
\begin{aligned}
B\left(G T^{+}, \omega\right)= & \sum_{\omega_{K}}\left[\left\langle\omega_{K=0}\left|\sigma_{0} t^{+}\right| 0\right\rangle^{2} \delta\left(\omega_{K=0}-\omega\right)\right. \\
& \left.+2\left\langle\omega_{K=1}\left|\sigma_{1} t^{+}\right| 0\right\rangle^{2} \delta\left(\omega_{K=1}-\omega\right)\right]
\end{aligned}
$$

in $\left[g_{A}^{2} / 4 \pi\right]$ units. The strength distributions will be referred to the excitation energy in the daughter nucleus, which are given by

$$
E_{e x}=\omega-E_{\pi_{0}}-E_{\nu_{0}},
$$

where $E_{\pi_{0}}$ and $E_{\nu_{0}}$ are the lowest quasiparticle energies for protons and neutrons, respectively.

To describe odd- $A$ nuclei, I follow the usual strategy of blocking the state corresponding to a given $J^{\pi}$ and using the equal filling approximation to calculate its nuclear structure [31]. This approximation has been compared with other more sophisticated approaches, showing that it is sufficiently precise for most practical applications [42]. A microscopic justification has been given in terms of standard procedures of quantum statistical mechanics [43]. In principle, the blocked state is selected to minimize the energy among the states in the vicinity of the Fermi level. In cases where the $J^{\pi}$ of the nucleus is experimentally known, the natural option is to choose $J^{\pi}$ according to this value. In all the test cases studied later, these states appear always close to the Fermi level, as expected. In SHN, where there is no experimental information on $J^{\pi}$, the state $J^{\pi}$ that corresponds to the ground state is used, but several choices for them among the states that are close to the Fermi level are also used for comparison. Studying the sensitivity of the half-lives to the choice of $J^{\pi}$ is interesting because slight changes in the theoretical treatment may lead to different $J^{\pi}$ for the ground sates.

The GT strength distributions and $\beta$-decay half-lives have been studied in the past within this model in various mass regions that include neutron-deficient isotopes in the $A \approx 70$ mass region [44, 45] and in the lead region 39, 46, 47]; neutron-rich isotopes in medium-mass 48 51], and rare-earth nuclei [52]; and $f p$-shell nuclei 53 55]. The sensitivity of the GT strength distributions to different ingredients of the theoretical formalism were studied in those works with especial emphasis on the deformation dependence of the decay properties. In particular, the sensitivity of the energy distribution of the 
GT strength to deformation has been exploited to determine the nuclear shape by comparing theoretical results with $\beta$-decay measurements using the total absorption spectroscopy technique [56].

\section{B. Phase-space factors}

In $\beta^{+} / E C$ decay, the phase-space factors $f^{\beta^{+} / E C}\left(Z, W_{0}\right)$ contain two parts, positron emission and electron capture. The former, $f^{\beta^{+}}$, is computed numerically for each value of the energy including screening and finite size effects, as explained in Ref. [57],

$$
f^{\beta^{+}}\left(Z, W_{0}\right)=\int_{1}^{W_{0}} p W\left(W_{0}-W\right)^{2} \lambda^{+}(Z, W) \mathrm{d} W,
$$

with

$$
\lambda^{+}(Z, W)=2(1+\gamma)(2 p R)^{-2(1-\gamma)} e^{-\pi y} \frac{|\Gamma(\gamma+i y)|^{2}}{[\Gamma(2 \gamma+1)]^{2}},
$$

where $\gamma=\sqrt{1-(\alpha Z)^{2}}, y=\alpha Z W / p, \alpha$ is the fine structure constant, and $R$ is the nuclear radius. $W$ is the total energy of the $\beta$ particle, $W_{0}$ is the total energy available in $m_{e} c^{2}$ units, and $p=\sqrt{W^{2}-1}$ is the momentum in $m_{e} c$ units.

The electron capture phase factors, $f^{E C}$, have also been included following Ref. [57]:

$$
f^{E C}=\frac{\pi}{2} \sum_{x} q_{x}^{2} g_{x}^{2} B_{x}
$$

where $x$ denotes the atomic sub-shell from which the electron is captured that includes $K$ - and $L$-orbits. $q$ is the neutrino energy, $g$ is the radial component of the bound state electron wave function at the nucleus, and $B$ stands for other exchange and overlap corrections [57].

\section{C. $Q_{E C}$ energies}

$\beta^{+} / E C$ half-lives depend critically on the $Q$-energies that determine the maximum energy of the transition and the values of the phase factors that weight the GT strength, see Eq. (2). In those cases where experimental masses are available [58, 59], the natural choice is to use these values to evaluate Eq. (11). But in those cases where experimental masses are still missing, one has to rely on theoretical predictions for them. There are a large number of mass formulas in the market obtained from different approaches. The strategy followed in this work starts by comparing with experiment the predictions of some representative mass formulas in the mass region where data are available and use them later in SHN where there is no experimental information.
Among the phenomenological approaches for the masses, I take the FRDM [26] that belongs to a macroscopic-microscopic type of calculation. It contains a finite-range droplet model corrected by microscopic effects obtained from a deformed single-particle model based on a folded-Yukawa potential including pairing in the Lipkin-Nogami approach. Then, I use the extended Thomas-Fermi plus Strutinsky integral (ETFSI) model [60], which adopts a semi-classical approximation to the Hartree-Fock method including full Strutinsky shell corrections and BCS pairing correlations. The Duflo and Zuker (DZ) mass model [61] is used as well, which is written as an effective Hamiltonian that contains monopole and multipole terms. I also compare with fully microscopic calculations based on effective two-body nucleonnucleon interactions. Among them, I consider the masses from the HFB-21 model, which is one of the most recent versions of the Skyrme HFB mass formulas introduced by the Brussels-Montreal group 62]. I also use the masses calculated from the Skyrme forces SkP and SLy4 with a zero-range pairing force and Lipkin-Nogami obtained from the code HFBTHO 63]. All the masses used here can be found in Ref. 64].

\section{RESULTS FOR THE HALF-LIVES}

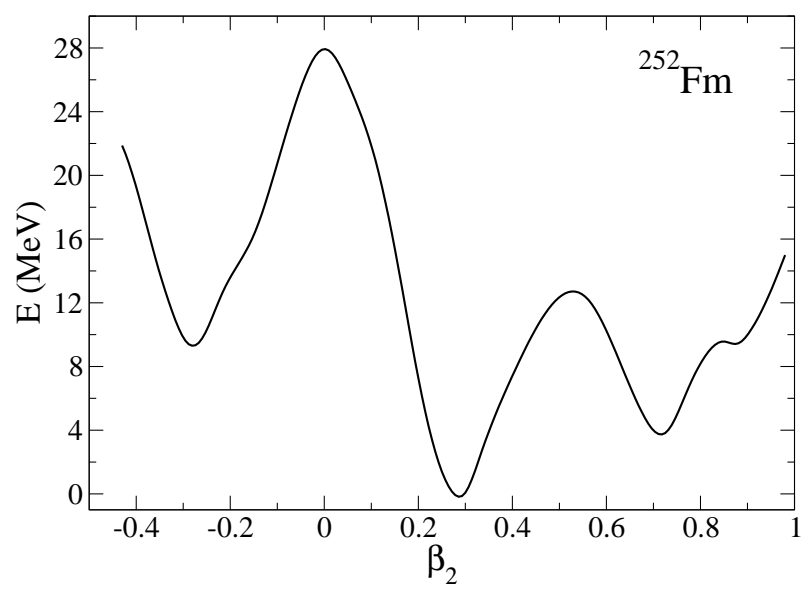

FIG. 1: Deformation-energy curve for the ${ }^{252} \mathrm{Fm}$ isotope obtained from constrained $\mathrm{HF}+\mathrm{BCS}$ calculations with the Skyrme force SLy4.

In this section I present the calculations for the halflives in SHN. I first show the results obtained for the DECs in the isotopes studied. The energy distributions of the GT strength corresponding to the local minima of the DECs are calculated afterwards. Finally, half-lives are computed.

Before starting with the calculations of the SHN mentioned in the Introduction, the quality of the calculations is checked in some isotopes around $Z=100$, where both $Q_{E C}$ and $T_{\beta^{+} / E C}$ have been measured. Namely, the isotopes of Fermium $(Z=100){ }^{246,247} \mathrm{Fm}$; Mendelevium 

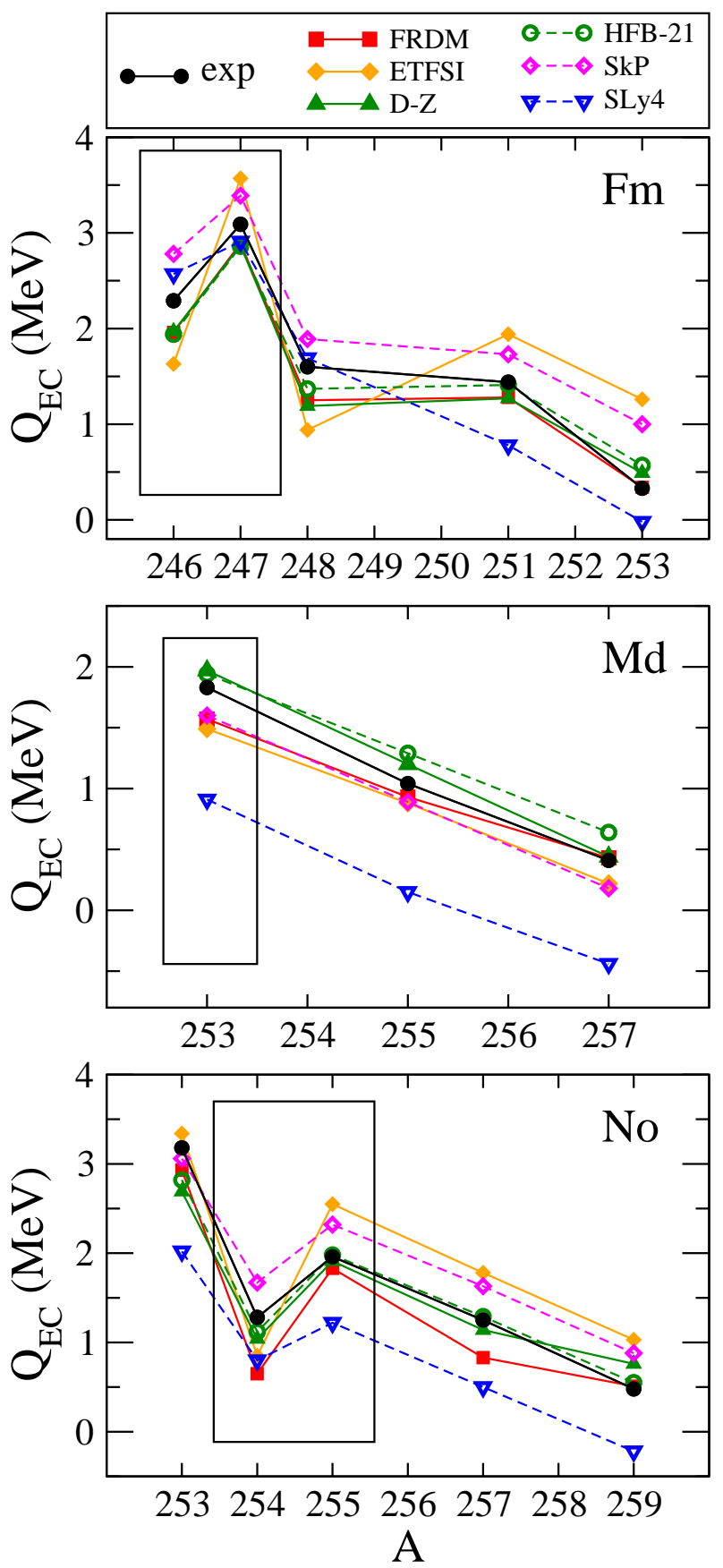

FIG. 2: $Q_{E C}$ energies $(\mathrm{MeV})$ corresponding to experimental and different calculated masses (see text) for $\mathrm{Fm}, \mathrm{Md}$, and No isotopes.

$(Z=101){ }^{253} \mathrm{Md} ;$ and Nobelium $(Z=102){ }^{254,255} \mathrm{No}$, are considered. After this test, the results for the SHN studied in this work, ${ }^{290} \mathrm{Fl},{ }^{293} \mathrm{Mc},{ }^{294} \mathrm{Lv}$, and ${ }^{295} \mathrm{Ts}$, will be shown.

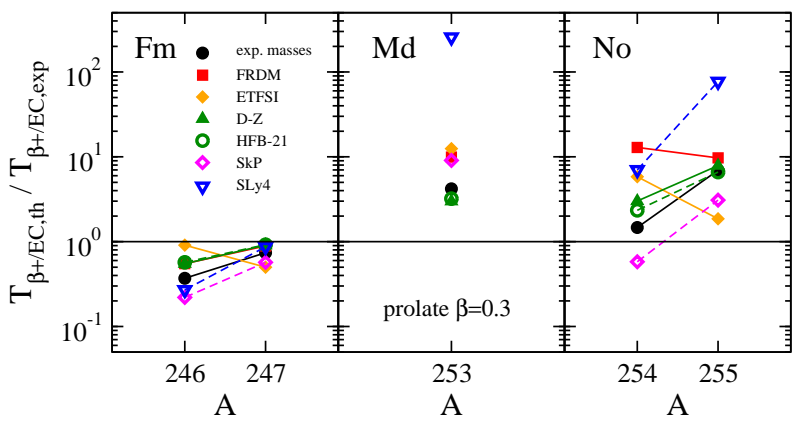

FIG. 3: Ratios of calculated and experimental half-lives for Fm, Md, and No isotopes. The results correspond to the ground state configurations (prolate $\beta_{2}=0.3$ ) using $Q_{E C}$ energies from different mass formulas or microscopic calculations.

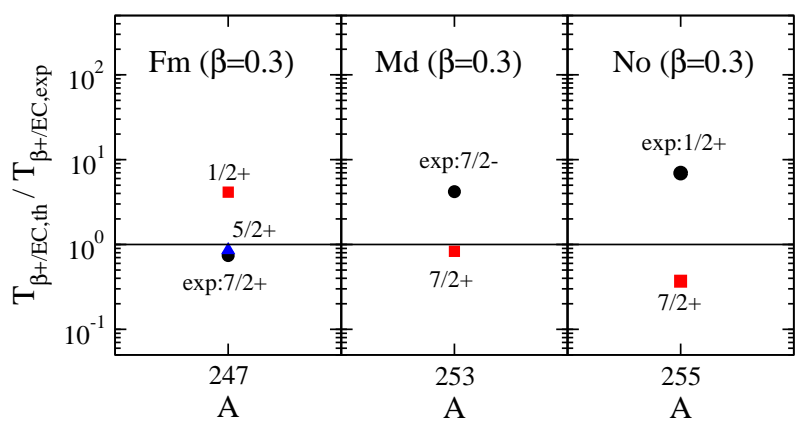

FIG. 4: Ratios of calculated and experimental half-lives for odd- $A \mathrm{Fm}, \mathrm{Md}$, and No isotopes. The results correspond to the ground state prolate configurations $\left(\beta_{2}=0.3\right)$, using the experimental $Q_{E C}$ energies and different $J^{\pi}$ assignments for the odd nucleon.

\section{A. Testing case: Fm, Md, and No isotopes}

Studying the deformation dependence of the energy by constrained calculations shows that nuclei in this region present three minima that correspond to oblate, prolate, and large prolate shapes. In Fig. 1 the DEC for ${ }^{252} \mathrm{Fm}$

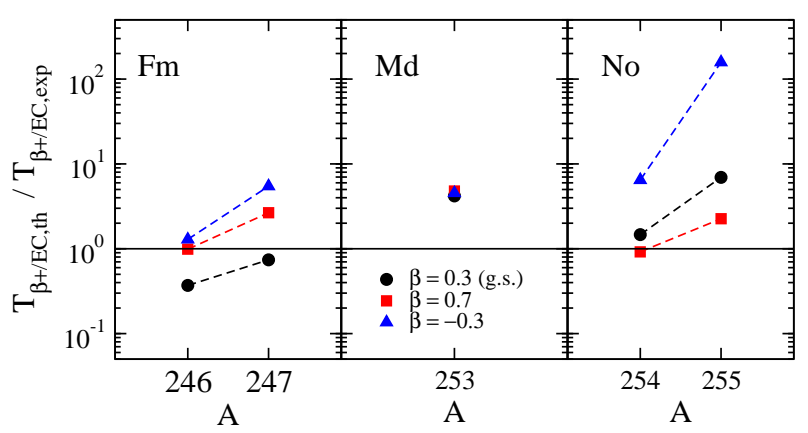

FIG. 5: Ratios of calculated and experimental half-lives for Fm, Md, and No isotopes. The results are obtained with experimental $Q_{E C}$ energies for the three shapes that produce energy minima in Fig. 1 . 

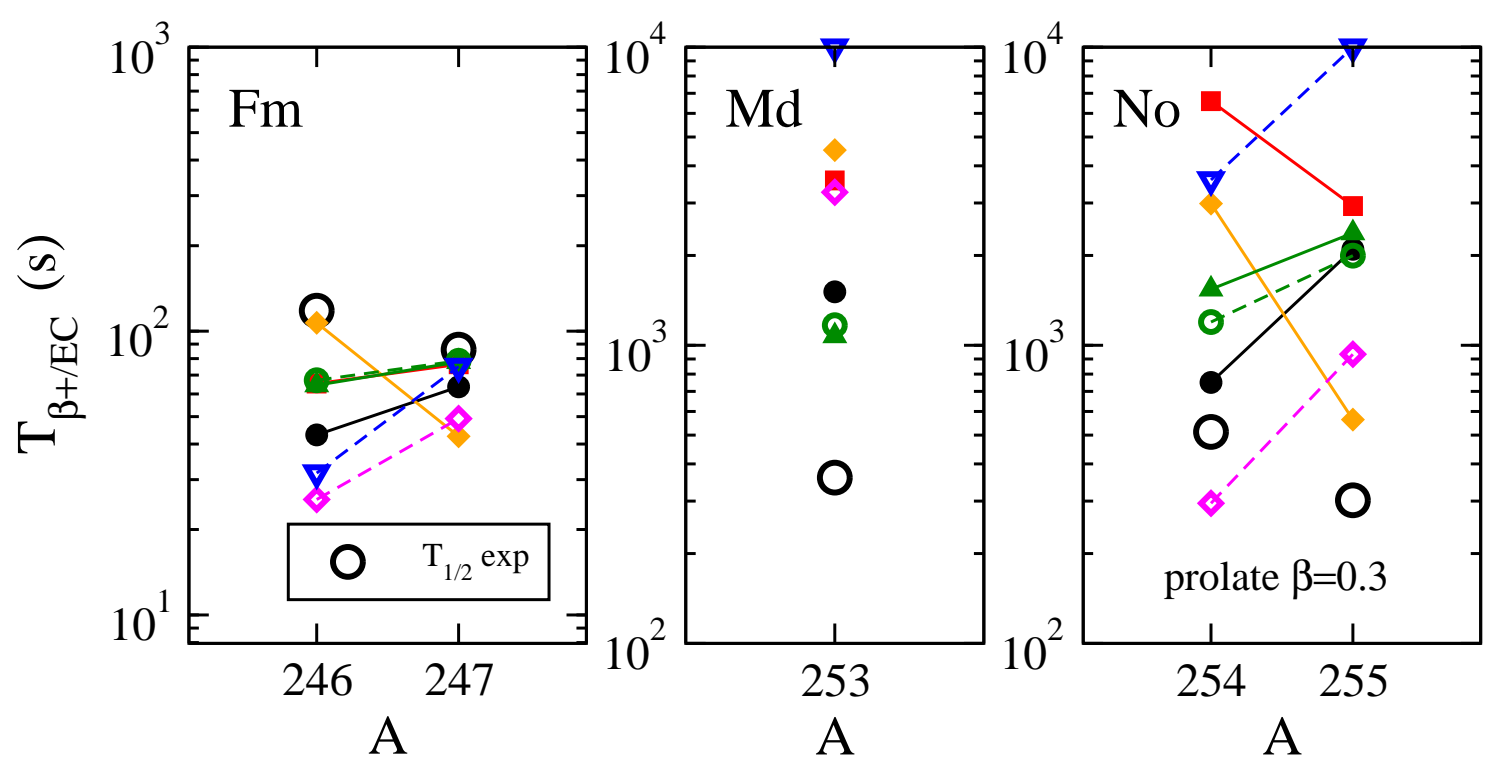

FIG. 6: Calculated and experimental half-lives for Fm, Md, and No isotopes. The results correspond to ground state configurations (prolate $\beta_{2}=0.3$ ) using different $Q_{E C}$ energies with the same code symbol of Fig. 3 .

with SLy4 is shown. The energies are relative to the ground state energy, as a function of the quadrupole deformation $\beta_{2}$. These results are very similar to the DECs for the other testing isotopes and I discuss only this case as an example. The ground state is found to have a prolate shape with a quadrupole deformation around $\beta_{2} \approx 0.3$, but there are also minima at oblate $\beta_{2} \approx-0.3$ and prolate $\beta_{2} \approx 0.7$ configurations at typical excitation energies around 8 and $4 \mathrm{MeV}$, respectively. These results agree quite well with calculations performed with the finite-range Gogny D1S interaction 65].

Experimental masses of parent and daughter nuclei in this mass region are available and then, one gets experimental $Q_{E C}$ energies. However, in the heavier nuclei considered in the next section, this information is missing and one has to rely on the predictions of mass formulas. I have considered some of the most commonly used formulas (or microscopically calculated masses) as they appear in the nuclearmasses.org web page 64]. They are FRDM, ETFSI, DZ, HFB-21, SkP, and SLy4, introduced in the previous section. Figure 2 shows the $Q_{E C}$ energies from experiment and from different mass formulas and illustrates the spreading of the $Q_{E C}$ energies. The cases for which half-lives have been calculated appear within a frame in Fig. 2. One can see that the results are scattered about $1 \mathrm{MeV}$ between the largest and smallest energies among the cases considered. Experimental values appear within these extreme values. This gives us a fair idea of the uncertainties expected. Although the uncertainty is not very large, these $Q$-values determine the energy range of excitations that contribute to the halflives, as well as the magnitude of the phase factors and as it will be seen in the next figures, the effect on the half-lives is important.

Figures [3 [5] show the ratios of the calculated half-lives to the experimental ones for some Fm, Md, and No isotopes, where there are experimental data. The experimental values have been extracted from the total halflives measured together with the percentage that corresponds to the $\beta^{+} / E C$ decay. Figure 3 shows the results for the ground states (prolate with $\beta_{2} \approx 0.3$ ) and using the experimental $J^{\pi}$ in the case of odd- $A$ nuclei. The various calculations correspond to the different $Q_{E C}$ values either from experiment or from calculated masses. The half-lives of Fm isotopes are underestimated, whereas the half-lives of $\mathrm{Md}$ and No isotopes are somewhat overestimated. One can see a clear correlation between the $Q_{E C}$ energies in Fig. 2 and the half-lives in Fig. 3, that is, half-lives decrease with increasing values of $Q_{E C}$.

The decay would be in principle from the ground state of the parent nucleus (that determines the shape and $J^{\pi}$ in odd- $A$ nuclei), but I also performed calculations of $\beta^{+} / E C$ half-lives that correspond not only to the ground states, but also to other shapes and $J^{\pi}$. This helps us to understand the sensitivity of the results to different factors arising from various uncertainties.

Figure 4 shows the sensitivity of the half-lives to the $J^{\pi}$ assignments in odd- $A$ nuclei. The results are for the ground state shapes and experimental $Q_{E C}$ values. The odd states are chosen according to the experimental spin and parity, as well as other possibilities for states that appear very close to the Fermi level. In Fig. 5 lone can see the results obtained with experimental $Q_{E C}$ values, but for different shapes that include the ground state $\left(\beta_{2} \approx\right.$ $0.3)$, as well as the oblate $\left(\beta_{2} \approx-0.3\right)$ and superdeformed prolate $\left(\beta_{2} \approx 0.7\right)$, depicted in Fig. 11. Finally, Fig. 6. compares the half-lives (seconds) measured with the calculated ones using the ground state deformations and different prescriptions for the $Q_{E C}$ energies. This figure is similar to Fig. 3 but for the absolute values. 
From the results for the half-lives, one can learn about the uncertainties associated with different aspects of the calculations. The uncertainties on the half-lives related to the $Q_{E C}$ energies, $J^{\pi}$ assignments, and nuclear shapes are comparable, spreading the results about one order of magnitude. The agreement with experiment is roughly within this order.

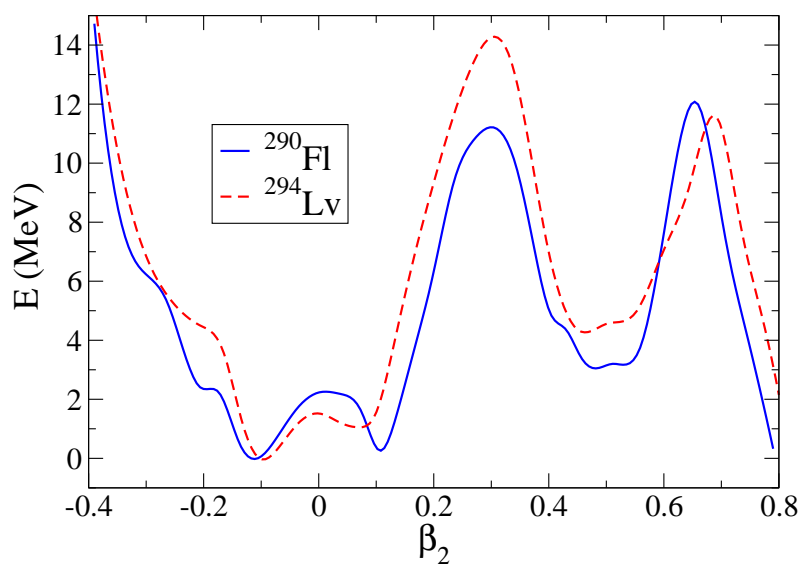

FIG. 7: Same as in Fig. 1, but for ${ }^{290} \mathrm{Fl}$ and ${ }^{294} \mathrm{Lv}$.

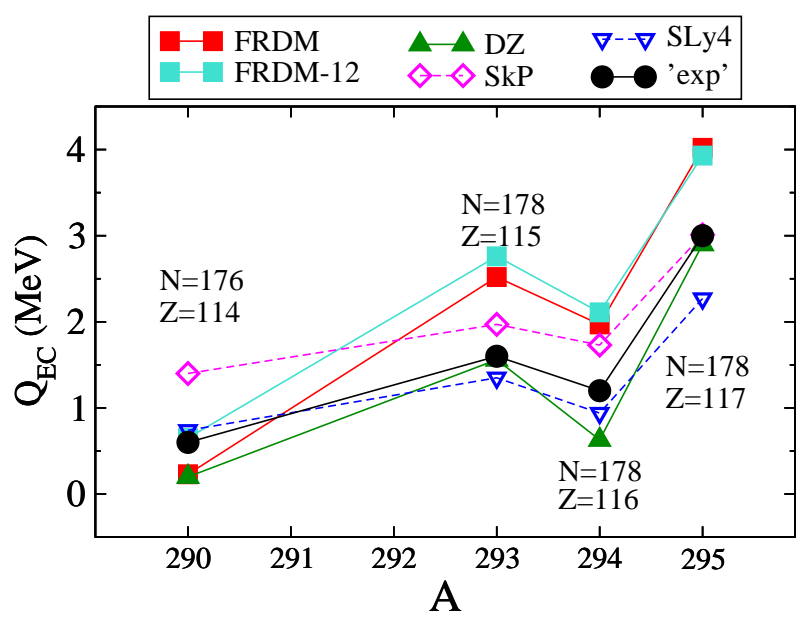

FIG. 8: $Q_{E C}$ energies $(\mathrm{MeV})$ corresponding to different calculated masses for SHN.

\section{B. Superheavy nuclei}

In this section the results for ${ }^{290} \mathrm{Fl},{ }^{293} \mathrm{Mc},{ }^{294} \mathrm{Lv}$, and ${ }^{295}$ Ts are discussed. In Fig. 7 the plots of the DECs are shown, relative to the ground state energy, for ${ }^{290} \mathrm{Fl}$ and ${ }^{294} \mathrm{Lv}$ as a function of the quadrupole deformation $\beta_{2}$ obtained from the Skyrme force SLy4. In both cases, the ground state is the oblate solution $\left(\beta_{2} \approx-0.1\right)$, while two more prolate minima appear at $\beta_{2} \approx 0.1$ and $\beta_{2} \approx 0.5$ at excitation energies of about $1 \mathrm{MeV}$ and $4 \mathrm{MeV}$, respectively. These results agree with those obtained from the Gogny-D1S interaction 65].

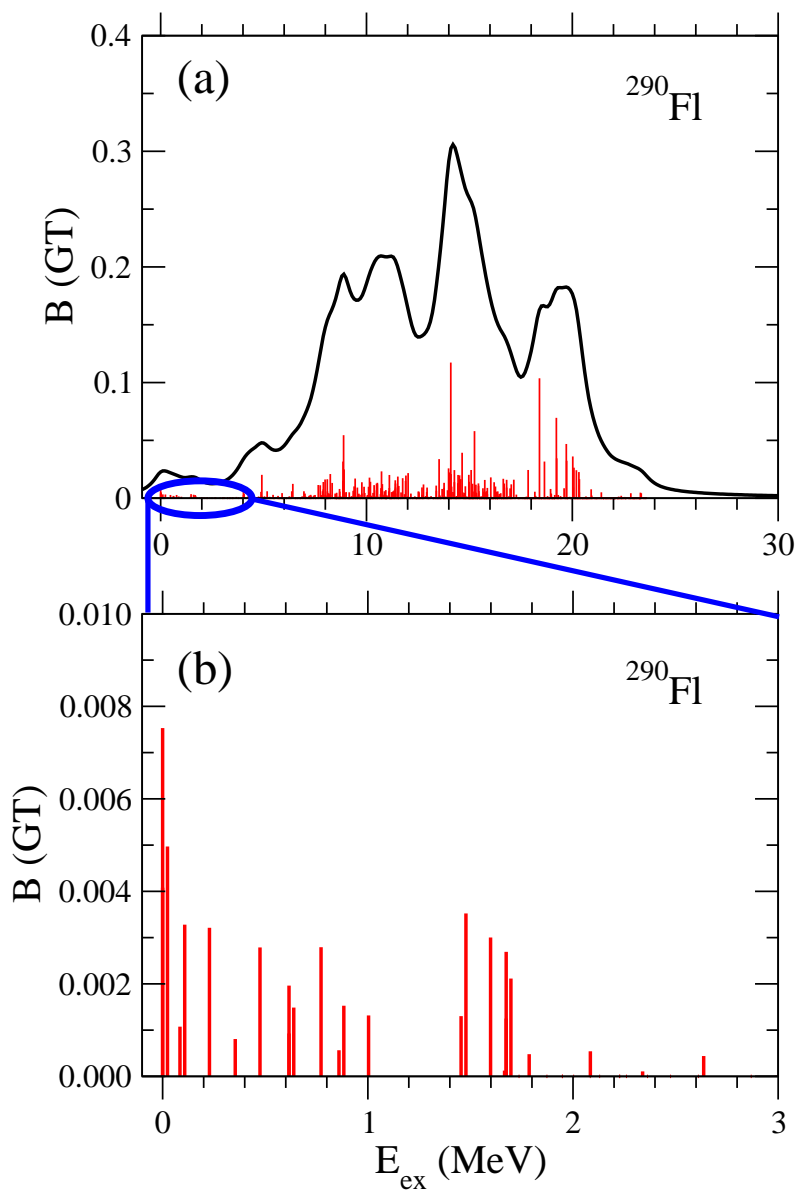

FIG. 9: (a) Gamow-Teller strength distribution in ${ }^{290} \mathrm{Fl}$ in the whole range of excitation energies of the daughter nucleus. (b) Magnified region below $3 \mathrm{MeV}$ that includes the $Q_{E C}$ energy.

Figure 8 shows the $Q_{E C}$ energies calculated with masses obtained from three mass formulas (FRDM, FRDM-12, and DZ) and two microscopic calculations (SkP and SLy4). The masses from FRDM-12 [66] are a recent improved upgrade of the FRDM masses. Although there are no experimental values for these nuclei, I also add extrapolated values from the systematics in this mass region extracted from Ref. [59], that appear in the figure under the label 'exp'. Similarly to the $Q_{E C}$ energies in Fig. 2, the results in Fig. 8 are distributed within $1 \mathrm{MeV}$ with the 'exp' values lying inside this range.

As a general comment, it is worth noting that the typical $Q_{E C}$ energies in these nuclei are rather small and, as a consequence, the half-lives are only sensitive to a very tiny part of the whole GT response of the nucleus. This means also that small changes in the nuclear structure description or in the $Q_{E C}$ energies, may produce very large effects on the half-lives. This is illustrated in Fig. 9 for ${ }^{290} \mathrm{Fl}$, where one can see the energy distribution of the GT strength in the whole range of energy (a) and below the $3 \mathrm{MeV}$ window (b), where the different mass models predict the $Q_{E C}$ energy. The half-life is only sensitive to the strength distribution in this small window. 

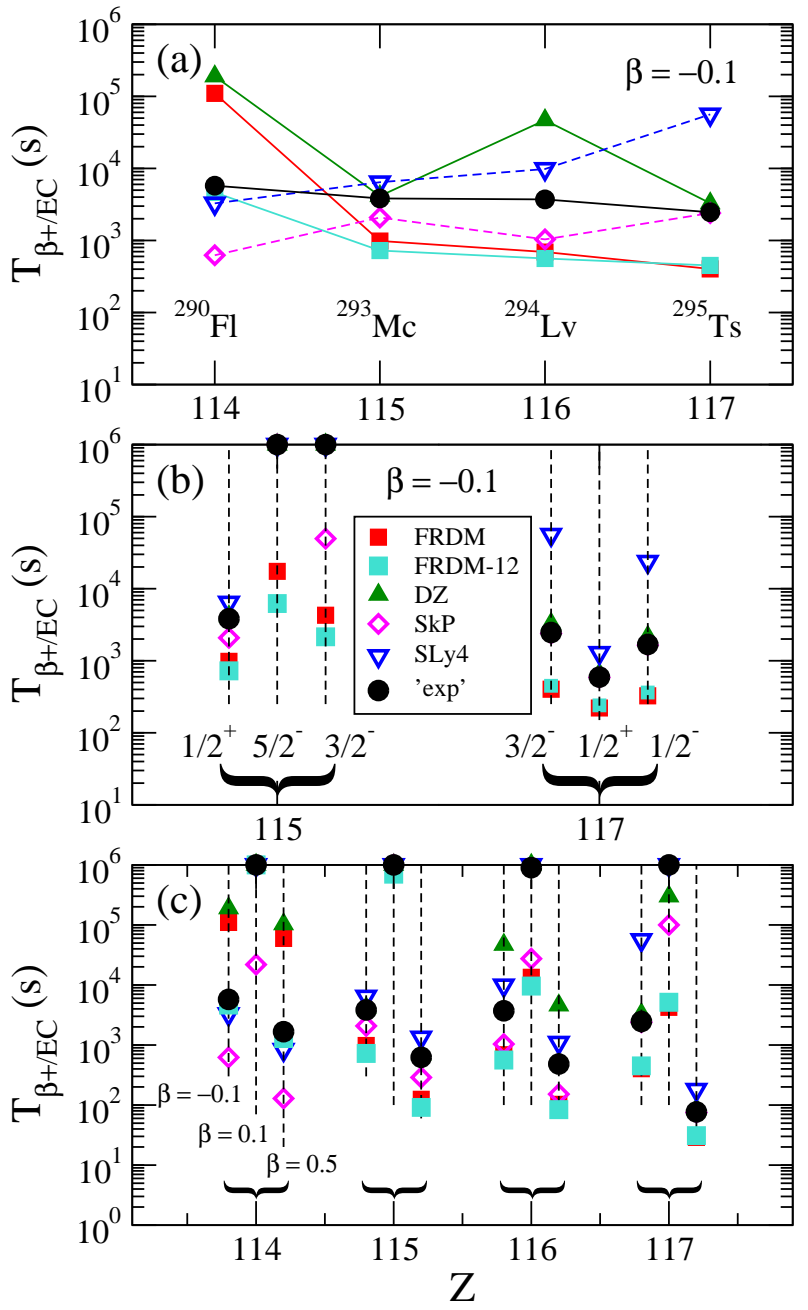

FIG. 10: Calculated half-lives, $T_{\beta}+E_{E C}(\mathrm{~s})$, for ${ }^{290} \mathrm{Fl},{ }^{293} \mathrm{Mc}$, ${ }^{294} \mathrm{Lv}$, and ${ }^{295} \mathrm{Ts}$. (a) Half-lives for ground state configurations (oblate $\beta_{2}=-0.1$ ) with various mass formulas to calculate $Q_{E C}$ energies. (b) Half-lives of odd- $A$ nuclei for ground state configurations (oblate $\beta_{2}=-0.1$ ) and several choices of $J^{\pi}$ values with various mass formulas to calculate $Q_{E C}$ energies. (c) Half-lives for oblate $\beta_{2}=-0.1$ (left vertical lines), prolate $\beta_{2}=0.1$ (middle vertical lines), and prolate $\beta_{2}=0.5$ (right vertical lines) nuclear shapes obtained from various mass formulas to calculate $Q_{E C}$.

The results for the $\beta^{+} / E C$-decay half-lives of the SHN are shown in Fig. 10. The top figure (a) summarizes the results. They correspond to the half-lives for the ground state configuration (oblate $\beta_{2} \approx-0.1$ ). The states $J^{\pi}$ in the odd- $A$ nuclei are those that minimize the energy. The spreading of the results corresponds to the different $Q_{E C}$ prescriptions and there is a clear correlation between the $Q_{E C}$ energies in Fig. 8 and the half-lives obtained with them. Thus, the large values of $Q_{E C}$ with the masses from FRDM and SkP make the half-lives shorter, whereas DZ and SLy4 having smaller $Q_{E C}$ energies, lead to larger half-lives. The half-lives obtained with the $Q_{E C}$ energies extrapolated from the experimental energies in neighbor nuclei appear around the average values.

In the middle figure (b) one can see the different results for odd- $A$ nuclei, using other $J^{\pi}$ states, which are also close to the Fermi level. The ground state of ${ }^{293} \mathrm{Mc}$ $(Z=115)$ corresponds to a $1 / 2^{+}$state that originates from the $i_{13 / 2}$ spherical orbital and there are two states very close in energy that correspond to $5 / 2^{-}$from $f_{5 / 2}$ and $3 / 2^{-}$from $p_{3 / 2}$. Similarly, the ground state of ${ }^{295} \mathrm{Ts}$ $(Z=117)$ corresponds to a $3 / 2^{-}$state whose origin is at $p_{3 / 2}$ spherical orbital, while two states very close in energy appear at $1 / 2^{+}\left(i_{13 / 2}\right)$ and $1 / 2^{-}\left(p_{3 / 2}\right)$. The sensitivity of the results to the $J^{\pi}$ assumed in the parent nucleus can be understood from the characteristics of the allowed transitions considered in this work. Allowed transitions correspond to $\Delta \pi=0$ and $\Delta J=0, \pm 1$ transitions and because of the small $Q_{E C}$ energies involved, only the low-lying excitations connecting the odd proton in $Z=115,117$ nuclei with neutron states in the vicinity of the Fermi level obeying the above selection rules are relevant. In the case of $Z=115$, it turns out that in the energy region around the neutron Fermi level, most of the states are positive-parity states and then, transitions from a proton $1 / 2^{+}$states are favored, whereas transitions from $3 / 2^{-}$and $1 / 2^{-}$states are suppressed. In the case of $Z=117$ the situation is similar, but some $1 / 2^{-}$neutron states are now present close to the neutron Fermi level. Then, although transitions from $1 / 2^{+}$ states are still stronger, decays from $1 / 2^{-}$and $3 / 2^{-}$are not so different. This explains why the half-lives of the positive-parity states $\left(1 / 2^{+}\right)$are smaller than those of the negative-parity states.

In the bottom plot (c) one can see the results corresponding to the oblate $\left(\beta_{2} \approx-0.1\right)$ in the left vertical lines, prolate $\left(\beta_{2} \approx 0.1\right)$ in the middle vertical lines, and superdeformed prolate $\left(\beta_{2} \approx 0.5\right)$ configurations in the right vertical lines for each nucleus. The deformations correspond to the minima of the DECs in Fig. 77 The half-lives obtained from the prolate shapes with $\beta_{2} \approx 0.1$ are in general larger than the ground state oblate values and then, they will not play any role in the decay. On the other hand, according to our calculations, the half-lives of the superdeformed shape isomers $\left(\beta_{2} \approx 0.5\right)$ are reduced by about one order of magnitude with respect to those of the ground states. The ground states of the superdeformed odd isotopes turn to be $9 / 2^{+}$states $\left(i_{13 / 2}\right)$, which are very different from the $J^{\pi}$ of the oblate ground states, thus favoring the shape isomeric possibility of these states. Using the extrapolated experimental $Q_{E C}$ energies, half-lives in the range of $100-1000 \mathrm{~s}$ in ${ }^{290} \mathrm{Fl},{ }^{293} \mathrm{Mc}$, and ${ }^{294} \mathrm{Lv}$ and around $100 \mathrm{~s}$ in ${ }^{295} \mathrm{Ts}$ are obtained for the superdeformed shapes. The possible decays from these shape isomers might compete with $\alpha$ decays, as will be seen in the next section. Because of the rather small excitation energies of these states of about $4 \mathrm{MeV}$ according to Fig. 7, they could be populated in hot-fusion reactions.

Summarizing these results, one can say that the $\beta^{+} / E C$-decay half-lives obtained for the $\mathrm{SHN}{ }^{290} \mathrm{Fl}$, 
${ }^{293} \mathrm{Mc},{ }^{294} \mathrm{Lv}$, and ${ }^{295} \mathrm{Ts}$, vary from $500 \mathrm{~s}$ up to $10^{5} \mathrm{~s}$, depending on the $Q_{E C}$ energies used. The average values around $10^{3}-10^{4} \mathrm{~s}$, are compatible with the the values obtained from the experimental extrapolated values for $Q_{E C}$. Decays from superdeformed shapes reduce the half-lives, making them comparable to $\alpha$-decays in some cases.

\section{C. $\alpha$-decay half-lives}

As already mentioned in the Introduction, the competition between the different decay modes is important to determine the pathways through stability and the SHN that can be reached from a given hot-fusion reaction. Therefore, I calculate in this section $\alpha$-decay half-lives to be compared with the corresponding $\beta^{+} / E C$-decay halflives of the previous section.

Similarly to the case of the $Q_{E C}$ energies discussed above, the $Q_{\alpha}$ energies can be obtained from the same mass evaluations used for $Q_{E C}$, using the expression

$$
Q_{\alpha}=M(A, Z)-M(A-4, Z-2)-M(4,2),
$$

written in terms of the nuclear masses $M(A, Z)$. In addition, I also include the values calculated in Ref. 21], obtained from a macroscopic-microscopic approach based on the two-center shell model applied to superheavy elements. There are no experimental measured values for these nuclei yet, but I quote in the last column the values obtained from extrapolation of the measured $Q_{\alpha}$ energies in neighboring nuclei [59]. These values are shown in Table【. They are representative of the most commonly used mass evaluations, but even more calculations of $Q_{\alpha}$ energies can be found in the review of Ref. 24], where values from models based on Woods-Saxon, $\mathrm{SkM}^{*}$, generator coordinate method, and relativistic Hartree-Bogoliubov are given as well. The values in Table प are in general agreement with those in Ref. 24].

TABLE I: $Q_{\alpha}$ energies (MeV) obtained from different mass models. The energies in the last column 'exp' correspond to an extrapolation of the experimental values [59].

\begin{tabular}{cccccccc}
\hline \hline Nucleus & FRDM & FRDM-12 & DZ & SkP & SLy4 & {$[21]$} & 'exp' \\
\hline${ }^{290} \mathrm{Fl}$ & 8.50 & 8.84 & 10.76 & 9.72 & 9.84 & 8.90 & 9.9 \\
${ }^{293} \mathrm{Mc}$ & 9.47 & 9.44 & 10.84 & 9.28 & 9.21 & - & 10.1 \\
${ }^{294} \mathrm{Lv}$ & 10.97 & 10.91 & 11.13 & 9.48 & 9.41 & 10.44 & 10.6 \\
${ }^{295} \mathrm{Ts}$ & 11.58 & 11.54 & 11.48 & 9.85 & 9.72 & 10.53 & 11.1 \\
\hline \hline
\end{tabular}

The $\alpha$-decay half-lives of these nuclei are not measured yet, but there exist in the literature phenomenological formulas that have been fitted in different mass regions and that can be very useful to see the systematics and to predict these values in other regions not yet measured. I present here four of these parametrizations, which are specifically designed to account for the properties of SHN. These are the following:

- The formula by Parkhomenko and Sobiczewski 67. (label 1 in the x-axis of Fig[11):

$\log _{10}\left(T_{\alpha}\right)=a Z\left(Q_{\alpha}-E_{\mu}\right)^{-1 / 2}+b Z+c$,

with $a=1.5372, b=-0.1607, c=-36.573$, $E_{\mu}($ even - even $)=0, E_{\mu}($ odd-proton $)=0.113 \mathrm{MeV}$.

- The formula by Royer [68] (label 2 in the x-axis of Fig[11):

$\log _{10}\left(T_{\alpha}\right)=a Z\left(Q_{\alpha}\right)^{-1 / 2}+b Z^{1 / 2} A^{1 / 6}+c$,

with parameters from Ref. [67] for even-even nuclei, $a=1.5519, b=-0.9761, c=-28.688$, and for odd-proton nuclei, $a=1.6070, b=-0.9467$, $c=-30.912$.

- The Viola-Seaborg formula [69] (labels 3 and 4 in the $\mathrm{x}$-axis of Fig 111):

$$
\log _{10}\left(T_{\alpha}\right)=(a Z+b)\left(Q_{\alpha}\right)^{-1 / 2}+(c Z+d)+h_{i} .
$$

Two different sets of parameters are used for this formula:

(label 3) 67], $a=1.3892, b=13.862, c=-0.1086$, $d=-41.458, h_{e e}=0, h_{\text {odd-proton }}=0.437$, and

(label 4) [22, 70], $a=1.66175, b=-8.5166, c=$ $-0.20228, d=-33.9069, h_{e e}=0, h_{\text {odd-proton }}=$ 0.772 .

Figure 11] shows the $\alpha$-decay half-lives $T_{\alpha}(\mathrm{s})$ for ${ }^{290} \mathrm{Fl}$, ${ }^{293} \mathrm{Mc},{ }^{294} \mathrm{Lv}$, and ${ }^{295} \mathrm{Ts}$. The results correspond to the seven different choices for $Q_{\alpha}$ given in Table 【 and four different options for phenomenological formulas of $T_{\alpha}$, labeled from 1 up to 4 in the $\mathrm{x}$-axis as explained above. These results agree with similar calculations performed in Refs. [24, 25].

From this figure one can see that, whereas phenomenological formulas for $T_{\alpha}$ give quite similar results, a strong dependence on the $Q_{\alpha}$ energies is found. $T_{\alpha}$ can vary as much as five orders of magnitude (even more in ${ }^{290} \mathrm{Fl}$ ) due to the uncertainties in $Q_{\alpha}$.

Phenomenological mass formulas, such as DZ and FRDM, have a tendency to predict short values of $T_{\alpha}$, which is a consequence of the large $Q_{\alpha}$ values (see Table I). The exception is FRDM in ${ }^{290} \mathrm{Fl}$ that predicts the largest $T_{\alpha}$ value. On the other hand, microscopic meanfield calculations with Skyrme forces (SkP and SLy4) predict larger $T_{\alpha}$ values in these nuclei. The $T_{\alpha}$ obtained from the macroscopic-microscopic approach of Ref. 21] are close to the half-lives calculated from the inferred experimental values. They represent a kind of average value that can be taken as a reference value to compare with the $T_{\beta}$ decays. Thus, $T_{\alpha}$ half-lives of the order of $10 \mathrm{~s}$ are expected in ${ }^{290} \mathrm{Fl}$, from 1 to $10 \mathrm{~s}$ in ${ }^{293} \mathrm{Mc}$, from 0.1 to 1 


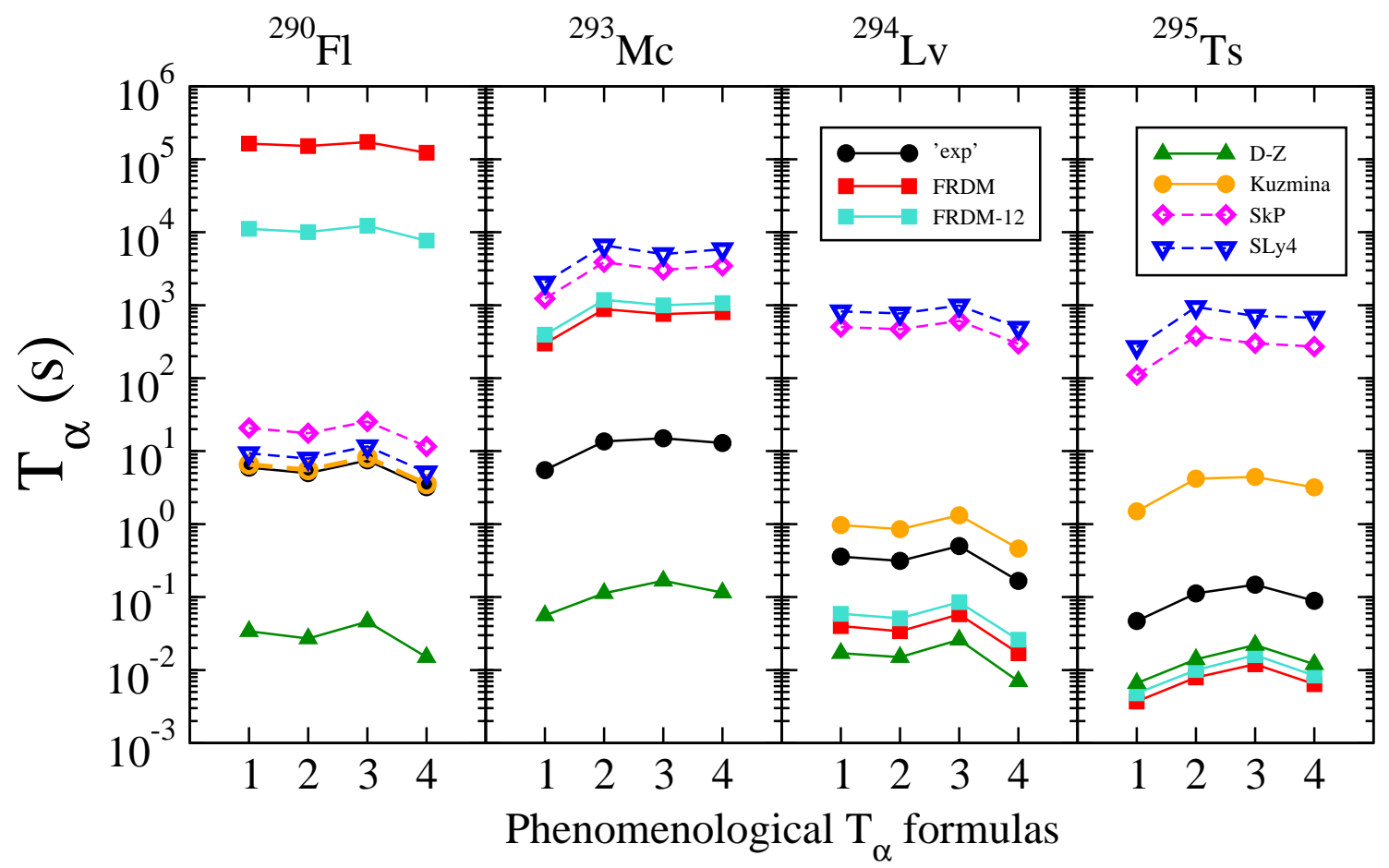

FIG. 11: $\alpha$-decay half-lives, $T_{\alpha}$ (s), for ${ }^{290} \mathrm{Fl},{ }^{293} \mathrm{Mc},{ }^{294} \mathrm{Lv}$, and ${ }^{295} \mathrm{Ts}$. The calculations correspond to six different choices for $Q_{\alpha}$ values, which are labeled in the figure, and four different options for phenomenological formulas of $T_{\alpha}$, labeled from 1 up to 4 in the $\mathrm{x}$-axis (see text).

$\mathrm{s}$ in ${ }^{294} \mathrm{Lv}$, and from 0.01 to $1 \mathrm{~s}$ in ${ }^{295} \mathrm{Ts}$. These values are always lower than the corresponding $T_{\beta^{+}} / E C$ halflives, and therefore $\beta^{+} / E C$ decay would be much slower than $\alpha$ decay in these nuclei, not competing with them. Only the $\beta^{+} / E C$ decay from superdeformed shapes with $T_{\beta^{+} / E C}$ half-lives around $10-100 \mathrm{~s}$ could have a chance to compete with $\alpha$ decay.

\section{SUMMARY AND CONCLUSIONS}

In this paper $\beta^{+} / E C$-decay half-lives in ${ }^{290} \mathrm{Fl},{ }^{293} \mathrm{Mc}$, ${ }^{294} \mathrm{Lv}$, and ${ }^{295} \mathrm{Ts}$, which are representative of SHN created in hot-fusion reactions, have been calculated microscopically. The calculations are based on a deformed Skyrme HF+BCS approach.

The uncertainties in the $\beta^{+} / E C$-decay half-lives that originate from poorly known $Q$-energies and $J^{\pi}$ assignments, as well as the influence of deformation, have been studied. The results are compared with $\alpha$-decay half-lives obtained from phenomenological parametrizations using the same mass formulas to determine the $Q_{\alpha}$ values.

Taking into account all the uncertainties in the results from both $\alpha$ and $\beta^{+} / E C$ decays, it is found that the latter are much larger than the former and therefore, there is in general no room for $\beta^{+} / E C$ decay to play a role in the decays of SHN produced in these hot-fusion reactions. The only possibility for a competition between both modes of decay would be the decay from superdeformed shape isomers that might be populated in the reactions.

\section{Acknowledgments}

I would like to thank G. Adamian for calling my attention to this problem, as well as for valuable discussions and a careful reading of the manuscript. This work was supported by Ministerio de Ciencia, Innovación y Universidades under Contract No. PGC2018-093636-B-I00.
[1] S. Hofmann and G. Münzenberg, Rev. Mod. Phys. 72, 733 (2000).

[2] Yu. Ts. Oganessian, V. K. Utyonkov, Yu. V. Lobanov, F. Sh. Abdullin, A. N. Polyakov, I. V. Shirokovsky, Yu. S. Tsyganov, G. G. Gulbekian, S. L. Bogomolov, A. N.
Mezentsev, S. Iliev, V. G. Subbotin, A. M. Sukhov, A. A. Voinov, G. V. Buklanov, K. Subotic, V. I. Zagrebaev, M. G. Itkis, J. B. Patin, K. J. Moody, J. F. Wild, M. A. Stoyer, N. J. Stoyer, D. A. Shaughnessy, J. M. Kenneally, and R. W. Lougheed, Phys. Rev. C 69, 021601(R) (2004). 
[3] Yuri Oganessian, J. Phys. G: Nucl. Part. Phys. 34, R165 (2007).

[4] Yu. Ts. Oganessian and V. K. Utyonkov, Nucl. Phys. A 944, 62 (2015).

[5] J. H. Hamilton, D. Hofmann, and Y. T. Oganessian, Annu. Rev. Nucl. Part. Sci. 63, 383 (2013).

[6] Juhee Jong, G. G. Adamian, and N. V. Antonenko, Phys. Lett. B 764, 42 (2017).

[7] S. A. Giuliani, Z. Matheson, W. Nazarewicz, E. Olsen, P.- G. Reinhard, J. Sadhukhan, B. Schuetrumpf, N. Schunck, and P. Schwerdtfeger, Rev. Mod. Phys. 91, 011001 (2019).

[8] S. Hofmann, S. Heinz, R. Mann, J. Maurer, G. Münzenberg, S. Antalic, W. Barth, H.G. Burkhard, L. Dahl, K. Eberhardt, R. Grzywacz, J.H. Hamilton, R.A. Henderson, J.M. Kenneally, B. Kindler, I. Kojouharov, R. Lang, B. Lommel, K. Miernik, D. Miller, K.J. Moody, K. Morita, K. Nishio, A.G. Popeko, J.B. Roberto, J. Runke, K.P. Rykaczewski, S. Saro, C. Scheidenberger, H.J. Schött, D.A. Shaughnessy, M.A. Stoyer, P. ThörlePospiech, K. Tinschert, N. Trautmann, J. Uusitalo, and A.V. Yeremin, Eur. Phys. J. A 52, 180 (2016).

[9] W. D. Myers and W. J. Swiatecki, Nuclear Phys. 81, 1 (1966).

[10] A. Sobiczewski, F. A. Gareev, and B. N. Kalinkin, Phys. Lett. 22, 500 (1966).

[11] S. G. Nilsson, J. R. Nix, A. Sobiczewski, Z. Szymanski, S. Wycech, C. Gustafson, and P. Möller, Nucl. Phys. A 115, 545 (1968).

[12] Z. Patyk and A. Sobiczewski, Nucl. Phys. A 533, 132 (1991).

[13] P. Möller and J. R. Nix, J. Phys. G: Nucl. Part. Phys. 20, 1681 (1994).

[14] R. Smolanczuk, J. Skalski, and A. Sobiczewski, Phys. Rev. C 52, 1871 (1995).

[15] K. Rutz, M. Bender, T. Bürvenich, T. Schilling, P.-G. Reinhard, J. A. Maruhn, and W. Greiner, Phys. Rev. C 56, 238 (1997).

[16] A. T. Kruppa, M. Bender, W. Nazarewicz, P.-G. Reinhard, T. Vertse, and S. Ćwiok, Phys. Rev. C 61, 034313 (2000).

[17] M. Bender, W. Nazarewicz, and P.-G. Reinhard, Phys. Lett. B 515, 42 (2001).

[18] M. Bender, P.-H. Heenen, and P.-G. Reinhard, Rev. Mod. Phys. 75, 121 (2003).

[19] J. Meng, H. Toki, S. G. Zhou, S. Q. Zhang, W. H. Long, and L. S. Geng, Prog. Part. Nucl. Phys. 57, 470 (2006).

[20] J. Dobaczewski, A. V. Afanasjev, M. Bender, L. M. Robledo, and Yue Shi, Nucl. Phys. A 944, 388 (2015).

[21] A. N. Kuzmina, G. G. Adamian, N. V. Antonenko, and W. Scheid, Phys. Rev. C 85, 014319 (2012).

[22] A. V. Karpov, V. I. Zagrebaev, Y. Martinez Palazuela, L. Felipe Ruiz, and Walter Greiner, Int. J. Mod. Phys. E 21, 1250013 (2012).

[23] V. I. Zagrebaev, A. V. Karpov, and Walter Greiner, Phys. Rev. C 85, 014608 (2012).

[24] P.-H. Heenen, J. Skalski, A. Staszczakc, and D. Vretenar, Nucl. Phys. A 944, 415 (2015).

[25] P. Möller, J. R. Nix, and K.-L. Kratz, At. Data Nucl. Data Tables 66, 131 (1997).

[26] P. Möller, J. R. Nix, W. D. Myers, and W. J. Swiatecki, At. Data Nucl. Data Tables 59, 185 (1995).

[27] E. O. Fiset and J. R. Nix, Nucl. Phys. A 193, 647 (1972),
[28] P. Sarriguren, E. Moya de Guerra, A. Escuderos, and A. C. Carrizo, Nucl. Phys. A 635, 55 (1998).

[29] P. Sarriguren, E. Moya de Guerra, and A. Escuderos, Nucl. Phys. A 658, 13 (1999).

[30] P. Sarriguren, E. Moya de Guerra, and A. Escuderos, Nucl. Phys. A 691, 631 (2001).

[31] P. Sarriguren, E. Moya de Guerra, and A. Escuderos, Phys. Rev. C 64, 064306 (2001).

[32] E. Chabanat, P. Bonche, P. Haensel, J. Meyer, and R. Schaeffer, Nucl. Phys. A 635, 231 (1998).

[33] M. Bender, G. F. Bertsch, and P.-H. Heenen, Phys. Rev. C 78, 054312 (2008).

[34] M. V. Stoitsov, J. Dobaczewski, W. Nazarewicz, S. Pittel, and D. J. Dean, Phys. Rev. C 68, 054312 (2003).

[35] D. Vautherin and D. M. Brink, Phys. Rev. C 5, 626 (1972); D. Vautherin, Phys. Rev. C 7, 296 (1973).

[36] M. V. Stoitsov, W. Nazarewicz, and S. Pittel Phys. Rev. C 58, 2092 (1998); M. V. Stoitsov, J. Dobaczewski, P. Ring, and S. Pittel, Phys. Rev. C 61, 034311 (2000).

[37] J. Krumlinde and P. Möller, Nucl. Phys. A 417, 419 (1984).

[38] H. Homma, E. Bender, M. Hirsch, K. Muto, H. V. Klapdor-Kleingrothaus, and T. Oda, Phys. Rev. C 54, 2972 (1996).

[39] J. M. Boillos and P. Sarriguren, Phys. Rev. C 91, 034311 (2015).

[40] K. Muto, E. Bender, T. Oda, and H. V. KlapdorKleingrothaus, Z. Phys. A 341, 407 (1992).

[41] A. Bohr and B. Mottelson, Nuclear Structure, Vols. I and II, (Benjamin, New York 1975).

[42] N. Schunck, J. Dobaczewski, J. McDonnell, J. Moré, W. Nazarewicz, J. Sarich, and M. V. Stoitsov, Phys. Rev. C 81, 024316 (2010).

[43] S. Perez-Martin and L. M. Robledo, Phys. Rev. C 78, 014304 (2008).

[44] P. Sarriguren, R. Alvarez-Rodríguez, and E. Moya de Guerra, Eur. Phys. J. A 24, 193 (2005).

[45] P. Sarriguren, Phys. Rev. C 79, 044315 (2009); Phys. Lett. B 680, 438 (2009); Phys. Rev. C 83, 025801 (2011).

[46] P. Sarriguren, O. Moreno, R. Alvarez-Rodríguez, and E. Moya de Guerra, Phys. Rev. C 72, 054317 (2005).

[47] O. Moreno, P. Sarriguren, R. Alvarez-Rodríguez, and E. Moya de Guerra, Phys. Rev. C 73, 054302 (2006).

[48] P. Sarriguren and J. Pereira, Phys. Rev. C 81, 064314 (2010).

[49] P. Sarriguren, A. Algora, and J. Pereira, Phys. Rev. C 89, 034311 (2014).

[50] P. Sarriguren, Phys. Rev. C 91, 044304 (2015).

[51] P. Sarriguren, A. Algora, and G. Kiss, Phys. Rev. C 98, 024311 (2018).

[52] P. Sarriguren, Phys. Rev. C 95, 014304 (2017).

[53] P. Sarriguren, E. Moya de Guerra, and R. AlvarezRodríguez, Nucl. Phys. A 716, 230 (2003).

[54] P. Sarriguren, Phys. Rev. C 87, 045801 (2013).

[55] P. Sarriguren, Phys. Rev. C 93, 054309 (2016).

[56] E. Nácher, A. Algora, B. Rubio, J. L. Taín, D. CanoOtt, S. Courtin, Ph. Dessagne, F. Maréchal, Ch. Miehé, E. Poirier, M. J. G. Borge, D. Escrig, A. Jungclaus, P. Sarriguren, O. Tengblad, W. Gelletly, L. M. Fraile and G. Le Scornet, Phys. Rev. Lett. 92, 232501 (2004).

[57] N.B. Gove and M.J. Martin, Nucl. Data Tables 10, 205 (1971).

[58] G. Audi, F. G. Kondev, M. Wang, B. Pfeiffer, X. Sun, J. Blachot, and M. MacCormick, Chinese Physics C 36, 
1157 (2012); M. Wang. G. Audi, A. H. Wapstra, F. G. Kondev, M. MacCormick, X. Xu, and B. Pfeiffer, Chinese Physics C 36, 1603 (2012).

[59] www.nndc.bnl.gov

[60] Y. Aboussir, J. M. Pearson, A. K. Dutta, F. Tondeur, At. Data Nucl. Data Tables 61, 127 (1995).

[61] J. Duflo and A. P. Zuker, Phys. Rev. C 52, R23 (1995).

[62] S. Goriely, N. Chamel, and J. M. Pearson, Phys. Rev. C 82, 035804 (2010).

[63] M. V. Stoitsov, J. Dobaczewski, W. Nazarewicz, and P. Ring, Comp. Phys. Comm. 167, 43 (2005).

[64] www.nuclearmasses.org

[65] S. Hilaire and M. Girod, Eur. Phys. J. A 33, 237 (2007); www-phynu.cea.fr/science_en_ligne/carte_potentiels_microscopiques/carte_potentiel_nucleaire_eng.htm

[66] P. Möller, A. J. Sierk, T. Ichikawa, and H. Sagawa, At. Data Nucl. Data Tables 109-110, 1 (2016).

[67] A. Parkhomenko and A. Sobiczewski, Acta Physica Polonica B 36, 3095 (2005).

[68] G. Royer, J. Phys. G: Nucl. Part. Phys. 26, 1149 (2000).

[69] V. E. Viola, Jr., and G. T. Seaborg, J. Inorg. Nucl. Chem. 28, 741 (1966).

[70] A. Sobiczewski, Z. Patyk, and S. Ćwiok, Phys. Lett. B 224, 1 (1989). 